\title{
OVERVIEW OF THE SPEAR3 VACUUM SYSTEM*
}

\author{
N. R. Kurita, R. M. Boyce, E. F. Daly, B. D. Scott, T. A. Trautwein
}

Stanford Synchrotron Radiation Laboratory, Stanford University, Stanford, CA 94309 USA

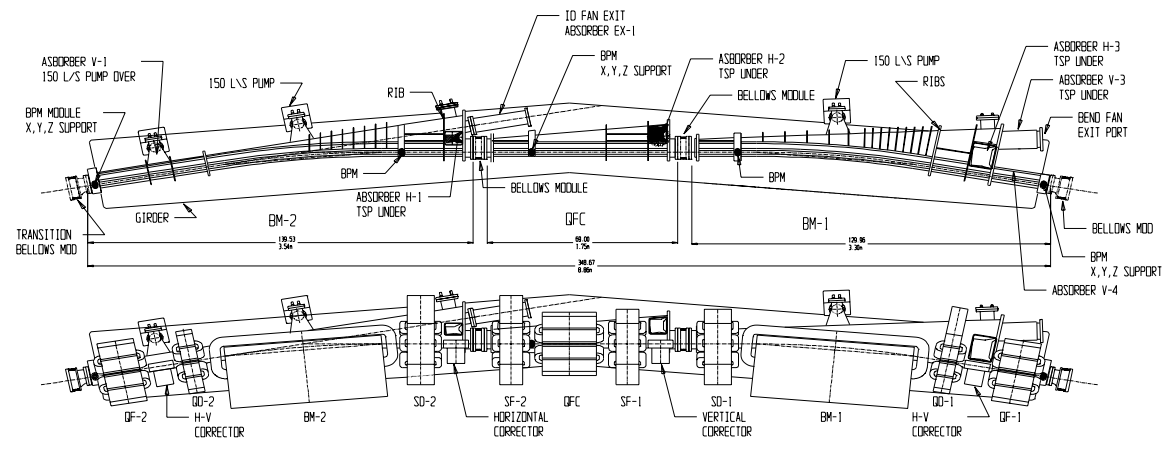

Figure 1: Standard Girder Layout

\section{Abstract}

The conceptual design of the vacuum system for the upgrade project of SPEAR3 is reviewed. The majority of the vacuum system components, except for insertion devices, will be replaced with components capable of $500 \mathrm{~mA}$ operation at 3 $\mathrm{GeV}$. General requirements and overall layout of the vacuum system are discussed. Diagnostic chambers and speciality components are briefly discussed.

\section{INTRODUCTION}

SPEAR3 is a $3 \mathrm{GeV}$ and $500 \mathrm{~mA}$ synchrotron light source. The magnet lattice was modified to an $18 \mathrm{~nm}$-rad double-bend achromat (DBA)[1]. The SPEAR3 ring is a racetrack oval $243 \mathrm{~m}$ in circumference, consisting of two arcs and two $6 \mathrm{~m}$ long straights. Each arc contains two $11 \mathrm{~m}$ matching girder chambers adjacent to the long straights and seven $9 \mathrm{~m}$ standard magnet girders joined by six $3 \mathrm{~m}$ straight sections. New vacuum chambers will be constructed except for the Insertion Device (ID) chambers. The standard girder layout is shown in figure 1 .

\section{GENERAL REQUIREMENTS}

SPEAR3 incorporates an antechamber design with discrete pumping and crotch absorbers. The vacuum chambers are designed to reach $500 \mathrm{~mA}$ at $3 \mathrm{GeV}$ and initial operation goals for FY 2002 is to achieve $200 \mathrm{~mA}$. To reach $500 \mathrm{~mA}$, ID chambers and beamline front ends will need to be upgraded. The desired lifetime during $500 \mathrm{~mA}$ operation is 40 hours.

The beam-stay-clear (BSC) envelope is defined for the entire vacuum system, excluding insertion devices, by the largest beam aperture in the ring. This occurs at the injection area and is modeled as an ellipse $30 \mathrm{~mm}$ high by $80 \mathrm{~mm}$ wide.[2] The interlock system trip level for high current operation will be set at $\pm 1 \mathrm{~mm}$ and a vertical emittance near $50 \mathrm{~nm} * \mathrm{rad}$. The trip level for the horizontal closed orbit shifts is set at $\pm 5 \mathrm{~mm}$. The interlock trip levels ensure that a mis-steered beam would not result in synchrotron radiation striking the vacuum chamber above or below the slot. The trip levels accommodate

Work supported by the U.S. Department of Energy under contract number DE-AC03-768F00515 alignment and manufacturing tolerances of the vacuum chambers and the magnets, as well as, deflection of the chambers and BPM accuracy. [2] The orbit shifts used for the design of the chamber are,

- Horizontal: $\pm 10 \mathrm{~mm}$ with $1.34 \mathrm{mrad}$ angular offset,

- Vertical: $\pm 6 \mathrm{~mm}$ with $0.8 \mathrm{mrad}$ angular offset.

\subsection{Steady State and Transient Thermal Loads}

During normal operation, the thermal loads due to resistive wall losses in SS are estimated to be $0.005 \mathrm{~W} / \mathrm{cm}^{2}$ and create less than a $10^{\circ} \mathrm{C}$ rise in the chamber. HOM losses are negligible. Axial cooling tubes with minimal flow removes this small amount of power.

During off-axis operation, the largest source of thermal loading is from ID SR impinging on the chamber walls, with a heat flux of $\sim 140 \mathrm{~W} / \mathrm{mm}^{2}$ from beamline 6 . The secondary source is SR emitted from the dipoles, approximately $20 \%$ of the maximum ID power. No practical amount of water cooling close to the strike can protect the chamber. At full power, the time-to-melt for $\mathrm{Cu}\left(\mathrm{MP}=1085^{\circ} \mathrm{C}\right)$ exceeds $500 \mathrm{~ms}$ and the time-to-melt for $\mathrm{SS}\left(\mathrm{MP}=1397^{\circ} \mathrm{C}\right)$ is less than $100 \mathrm{~ms}$.

\subsection{Masks and Absorbers}

The primary function of the discrete masks and absorbers is to shadow the chamber from dipole radiation. A distinction is made between absorbers and masks; absorbers are located in crotch areas between exit beamlines, masks are not.

\begin{tabular}{|l|c|c|c|}
\hline $\begin{array}{c}\text { Mask or } \\
\text { Absorber }\end{array}$ & $\begin{array}{c}\text { Ave. Fan } \\
\text { Ht }(\mathbf{m m})\end{array}$ & $\begin{array}{c}\text { Power } \\
(\mathbf{k W})\end{array}$ & $\begin{array}{c}\text { Heat Flux } \\
\left(\mathbf{W} / \mathbf{m m}^{2}\right)\end{array}$ \\
\hline Mask V-1 & 1.56 & 0.42 & 5.7 \\
\hline Mask V-2 & 2.42 & 0.53 & 2.4 \\
\hline Absorber H-1 & 0.48 & 4.76 & 21.5 \\
\hline Absorber H-2 & 0.94 & 3.92 & 5.5 \\
\hline Mask V-3 & 1.95 & 0.56 & 3.7 \\
\hline Absorber H-3 & 0.56 & 8.11 & 15.4 \\
\hline Mask V-4 & 0.43 & 1.95 & 19.3 \\
\hline
\end{tabular}

Table 1: Absorber \& Mask Heat Fluxes 
Three of the four masks are located along the outside of the chamber to intercept SR power. The total power incident on these three is only $500 \mathrm{~W}$. The surface that intercepts the power is a sheath of GlidCop ${ }^{\mathrm{TM}}$ brazed to a $\mathrm{Cu}$ cooling tube. The planar face on the sheath is oriented vertically and sloped at $30^{\circ}$ to grazing.

The fourth mask, V-4, located behind absorber $\mathrm{H}-3$, protects the downstream bellows module and chamber wall. The lineal heat flux on this GlidCop ${ }^{\mathrm{TM}} 7.5^{\circ}$-to-grazing surface is $83 \mathrm{~W} / \mathrm{cm}$.

The absorbers have crenulated surfaces that spreads out the intercepted power and are sloped at $10^{\circ}$-to-grazing relative to the horizontal plane. Water cooling is provided on the backside of the incident surface.

The exit aperture from the ring for all ID beamlines is $18 \mathrm{mrad}$. Three of the ID fans are nearly as wide as the aperture, and therefore are more likely to strike the masks or absorbers during horizontal mis-steering. The masks and absorbers cannot withstand these large power densities. An additional mask, EX1 , is installed in these locations and defines the $18 \mathrm{mrad}$ aperture for these specific beamlines. It is designed with a shallow angle-to-grazing to reduce the heat flux to acceptable levels.

The exit aperture for all insertion device beamlines is $18 \mathrm{mrad}$. The SR fan for three beamlines (4, 7 and 9) are nearly as wide as the aperture and are more likely to strike the masks and absorbers for small amounts of mis-steering. The power densities are about $\mathrm{X}$ times larger if ID power is intercepted on the $\mathrm{V}-1$ mask. The power density on $\mathrm{H}-1$ is $\mathrm{X}$ times greater, again unacceptable. The approach for exit ports is to have the mask and absorber define a $22 \mathrm{mrad}$ aperture, and then add an additional shallow angle surface beyond the exit port to then define the $18 \mathrm{mrad}$ aperture.

\section{GIRDER CHAMBER DESIGN}

The vacuum chamber cross section is a $34 \mathrm{~mm}$ high by 84 $\mathrm{mm}$ wide octagon with a $12 \mathrm{~mm}$ tall slot, see figure $\mathrm{x}$. This cross section allows a minimum of $\pm 2 \mathrm{~mm}$ between both the magnets and the prescribed BSC. The $2 \mathrm{~mm}$ clearance accommodates manufacturing and alignment tolerances, as well as the deflection of the chamber due to vacuum loading. The height of the slot is the most critical dimension of the vacuum chamber profile.

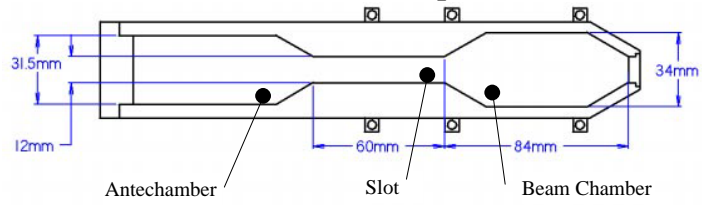

Figure 2: Typical Beam Cross Section, Copper Design.

Presently, two different designs are being pursued. One design is a Cu-coated, formed SS chamber similar to the ANKA and BESSY designs. The other is a machined $\mathrm{Cu}$ chamber incorporating PEP-II experience. Copper chambers will increase the passively safe dipole current of the machine from $20 \mathrm{~mA}$ for $\mathrm{SS}$ to $>500 \mathrm{~mA}$, increase the thermal response time to more than $500 \mathrm{~ms}$ and decrease resistive wall losses. The driving factors for material choice are cost and manufacturing risk.

\subsection{Stainless Steel Chamber}

Material Properties: SS has excellent vacuum properties, high mechanical strength, and is easy to weld. Rib placement is challenging but, they are necessary to keep the deflections of the chamber low especially at the slot. Since the deflections of the chamber are kept to a minimum the stresses due to vacuum loading are negligible. Optimization of rib geometry in ANSYS shows a $0.3 \mathrm{~mm}$ deflection can be achieved at the slot.

Eddy Currents: Orbit feedback is required for SPEAR3 to stabilize the beam orbit to less that $5 \mu \mathrm{m}$ [2]. Four corrector magnets on each girder are modulated as part of the orbit feedback scheme. Modulation of corrector elements in the lattice can create eddy currents within the vacuum chamber walls, inducing bucking fields that reduce the amplitude and shift the phase of the correction field. Primarily, the vertically modulated fields that provide horizontal beam correction are affected. SS has low electrical conductivity, thus circulating eddy currents are minimal. Initial calculations using MAFIA show acceptable field amplitudes for a $3 \mathrm{~mm}$ SS chamber with $1 \mathrm{~mm}$ of $\mathrm{Cu}$ plating. The calculations indicate that $\mathrm{Cu}$ compensating plates are required at the corrector locations to counteract the asymmetry created by the $\mathrm{Cu}$ coating on the slot.

Collective Effects: Resistive wall losses are high for a SS chamber. Beam instability calculations show that with a chromaticity of 0.1 the beam is stable up to $274 \mathrm{~mA}$. Therefore transverse feedback is not needed for initial commissioning, but may be necessary to achieve $500 \mathrm{~mA}$.

Manufacturing: The manufacturing process of the SS chambers is similar to ANKA and BESSY chambers. The chamber is formed from $3 \mathrm{~mm}$ SS into two halves, fired at 900 $\mathrm{C}$ to reduce hydrogen and to stress relieve, cleaned for UHV and EB welded. After final assembly of absorbers and pumps the chamber will be baked at $200 \mathrm{C}$.

A $\mathrm{Cu}$ layer $1 \mathrm{~mm}$ thick is thermally sprayed onto the SS chamber to increase the passively safe operating current for dipole power to $50 \mathrm{~mA}$ and to enable the brazing of $\mathrm{Cu}$ cooling tubes. Metallurgy and brazing tests are being performed on the sprayed $\mathrm{Cu}$. Also, to increase the passively safe operating current for insertion device power and thermal response time, a $\mathrm{Cu}$ insert was designed in the BM-2 chamber. The insert is machined from explosion bonded $\mathrm{Cu} / \mathrm{SS}$ transition material and EB welded into the chamber. With this addition the stainless chamber performs like a $\mathrm{Cu}$ chamber for ID power only.

\subsection{Copper Chamber}

Material Properties: Copper has excellent thermal properties that enable a more robust design when considering safe operating current and transient thermal response. The vacuum performance of $\mathrm{Cu}$ is nearly identical to SS for both thermal outgassing rates and photon stimulated desorption (PSD). The 
mechanical properties are more than adequate to achieve acceptable slot deflections and react vacuum loads as well as other loading conditions due to assembly.

Eddy Currents: Induced eddy currents can be significant in $\mathrm{Cu}$ due to its high electrical conductivity. By inserting a high resistance material in the loop, the effective circulating eddy current is reduced. MAFIA analysis has verified this approach. CuproNickel ${ }^{\mathrm{TM}}$ has an electrical conductivity about $1 / 20^{\text {th }}$ of $\mathrm{Cu}$ and can be welded reliably to $\mathrm{Cu}$. The design requires that all other conductive parts such as cooling tubes must not bridge the eddy current break.

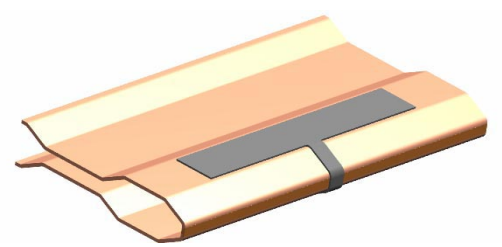

Figure 3: Chamber with CuNi Eddy Current Break

Collective Effects: Resistive wall losses for $\mathrm{Cu}$ are reduced by a factor of seven compared to SS, and potentially eliminate the need for a transverse feedback system. [3]

Manufacturing, Assembly and Processing: Copper chamber construction is divided into three major portions: machining of mating, mirror-image halves, and ancillary ports and flange preps, EB welding and assembly, and vacuum processing.

The upper and lower halves of each of the girder chambers are machined from single pieces of $\mathrm{OFE} \mathrm{Cu}$ plate. For the quantities required in SPEAR3, this approach falls within existing budgetary constraints.

After EB welding the chamber is required leak tight to $2 \times 10-10$ std cc/sec using Helium gas. The option for glow-discharge processing the beam passage with a 5\%Oxygen/95\%Argon gas mixture prior to bake-out is being considered to help reduce overall PSD rates. The chambers are then placed in vacuum ovens and processed at $200 \mathrm{C}$ until outgassing rates and partial pressures are below acceptable limits.

\section{STRAIGHT SECTION CHAMBERS}

The straight sections contain the ID, diagnostic and injection chambers, and RF cavities. SPEAR3 requires that the existing standard cell lengths and ID beam alignments be preserved. Also, all existing ID chambers will be kept, although they are only designed for $200 \mathrm{~mA}$. New masking is required for 500 mA operation.

Physics requirements dictate that the straight sections also maintain the BSC described previously. The same internal octagonal profile will be used for all new straight section chambers. Smooth transitions (1:5) are made between existing ID's where space permits.

Straight sections with no special chambers require two masks that intercept less than $10 \mathrm{~W} / \mathrm{cm}$ of SR power. Two refurbished ion pumps are mounted near the masks and additional pump ports may be added for future upgrades.
Straight section drift chambers are machined from copper and subsequently EB welded. Cu-to-SS adapters are used to weld on conflat flanges. Rectangular cooling tubes are welded directly to the chamber. This design allows a passively safe operating current well above $500 \mathrm{~mA}$.

Injection Kicker: The injection kicker, based on the proven DELTA design, consists of 2 conductors separated by four axial slots. The conductors produce an inductive loop and are connected to ground by metal bands. The design is electrically symmetric with two inputs for current pulses of opposite polarity. The slotted kicker pipe is $0.4 \mathrm{~m}$ long and is housed in a vacuum tank. The metal bands maintain the cross section, reducing impedance. Cooling channels inside of vacuum are required to cool a SR strike of $6.2 \mathrm{~W} / \mathrm{cm}$.

Transverse Kickers: Two $\mathrm{Cu}$ plates formed into partial ellipses are connected by Inconel flex supports to four $50 \Omega$ ceramic feedthrus that are welded to an elliptical vacuum chamber. Flex supports allow for the differential thermal expansion between the plates and the chamber.

The vertical kicker requires a $5 \mathrm{~mm}$ offset to shadow a direct SR strike, the horizontal does not. The primary mode of heat transfer is through radiation, because there is no significant conductive path to the vacuum chamber and internal cooling tubes could alter the performance of the kicker. A Cu-oxide using an arc deposition process developed at LBL for PEP-II is being investigated to blacken the electrodes.

Synchrotron Light Monitor (SLM): The SLM images visible and near ultraviolet light and is located in the $4.5 \mathrm{~m}$ matching straight section. The longer straight allows the primary mirror to be further away from its SR source, reducing the power density. The X-rays are concentrated in the vertical midplane of the radiation pattern, while the visible and UV light have much larger vertical opening angles and do not produce a significant amount of power. A thin mask intercepts the high power x-ray light and reduces thermal distortion of the primary mirror. The primary mirror is designed to withstand the compressive thermal stresses from an off-axis hit.

\section{BELLOWS MODULE}

The bellows module bridges the gap between the chambers and allows for thermal expansion, alignment, manufacturing tolerances and installation of the vacuum chambers. It also serves to isolate and stabilize the BPM's. SPEAR3 will use the double finger mechanism successfully developed at SLAC for PEP-II. It may be necessary to employ a mask to prevent the module from being destroyed by an off-axis SR strike.

\section{REFERENCES}

[1] Y. Nosochkov and J. Corbett, Dynamic Aperture Studies for SPEAR3 SLAC-PUB-7965, October 1998.

[2]SPEAR3 Conceptual Design Report, July 14, 1998.

[3] C. Limborg, J. Sebek, "Collective Effects in SPEAR3", US PAC99

Conference Proceedings, New York, New York. 